\title{
Dynamic object recognition in pigeons and humans
}

\author{
MARCIA L. SPETCH and ALINDA FRIEDMAN \\ University of Alberta, Edmonton, Alberta, Canada \\ and \\ QUOC C. VUONG \\ Max Planck Institute for Biological Cybernetics, Tübingen, Germany
}

\begin{abstract}
We investigated the role of dynamic information in human and pigeon object recognition. Both species were trained to discriminate between two objects that each had a characteristic motion, so that either cue could be used to perform the task successfully. The objects were either easy or difficult to decompose into parts. At test, the learned objects could appear in their learned motions, the reverse of the learned motions, or an entirely new motion, or a new object could appear in one of the learned motions. For humans, any change in the learned motion produced a decrement in performance for both the decomposable and the nondecomposable objects, but participants did not respond differentially to new objects that appeared in the learned motions. Pigeons showed the same pattern of responding as did humans for the decomposable objects, except that pigeons responded differentially to new objects in the learned motions. For the nondecomposable objects, pigeons used motion cues exclusively. We suggest that for some types of objects, dynamic information may be weighted differently by pigeons and humans.
\end{abstract}

Rapid and accurate recognition of objects in the environment can be critical for survival of humans and nonhumans alike, and hence the existence of cognitive processes necessary for object recognition should be widespread throughout the animal kingdom. Nevertheless, the nature of these processes could differ across species, making object recognition an interesting ability to investigate in comparative work. The visual and cognitive processes by means of which objects are recognized by shape information alone have been the subject of intensive investigation and theorizing in humans (e.g., Biederman, 1987; Bülthoff \& Edelman, 1992; Edelman, 1999; Tarr \& Pinker, 1989) and recently in pigeons (e.g., Friedman, Spetch, \& Ferrey, 2005; Peissig, Wasserman, Young \& Biederman, 2002; Spetch \& Friedman, 2003; Spetch, Friedman, \& Reid, 2001; Wasserman et al., 1996). Pigeons provide an interesting comparison with humans because they are highly visual creatures, but they differ substantially from humans both in their visual experiences and in the morphology of their visual system (see Husband \& Shimizu, 2001; Zeigler \& Bischof, 1993), each of which might impact object recognition. For example, birds, through flight, may require a different set of processes for rapid

This research was supported by grants from the Natural Sciences and Engineering Research Council of Canada and by the Max Planck Society. We are thankful to Bernd Kohler for help with programming the experiments, and to Alisha Brown, Emily Gray, Danielle Ludeman, Scott McAnsh, and Eric Verbeek for assistance with the research. Requests for reprints should be sent to M. L. Spetch, Department of Psychology, University of Alberta, Edmonton, AB, T6G 2E9 Canada (e-mail: mspetch@ualberta.ca). comprehension of the 3-D world than do humans. Pigeons also have two fovea-like specialized retinal areas, each of which is similar to the human fovea. One appears to be specialized for near frontal vision and presumably facilitates detection and selection of grain. The other area appears to serve more distant monocular lateral vision, and may allow navigation, approach and avoidance of objects during flying, and monitoring of predators (see Blough, 2001). Recent studies on recognition of static objects have suggested that there are interesting similarities and differences between the cognitive processes underlying the bird's eye view of the world and those that underlie our own (reviewed by Spetch \& Friedman, 2006).

More recently, researchers have begun to focus on the role that motion plays in object recognition, both in pigeons and in humans. Although motion is not the only source of visual changes in the environment, it is a prominent source of dynamic information. Some important objects are frequently viewed while they are in motion, such as an approaching predator or a fleeing prey. Such dynamic information could serve an important role in object recognition. On the one hand, dynamic information could facilitate the perception of shape information by allowing the detection and recovery of structure from motion (Wallach \& O'Connell, 1953). At the same time, dynamic information could provide a characteristic motion or spatiotemporal signature of the moving object that captures the manner in which shape information changes over time (Cavanagh, Labianca, \& Thornton, 2001; Stone, 1998; Vuong \& Tarr, 2006). For example, many animate objects have a characteristic motion that could assist in 
their identification, such as the slithering of a snake, the hopping of a grasshopper, or the fluttering of a butterfly.

There is good evidence that pigeons are sensitive to motion cues and can discriminate between different types of motion or different motion paths. For example, Bischof, Reid, Wylie, and Spetch (1999) showed that pigeons could discriminate coherent motion from incoherent motion in random dot displays, although their threshold for detecting coherence was somewhat higher than that of humans. Cook, Shaw, and Blaisdell (2001) have provided evidence that pigeons can discriminate between motion paths of objects. Specifically, pigeons learned to respond differentially according to whether the motion path of the camera's perspective moved through an object or moved around an object. Moreover, the discrimination transferred to new objects and was disrupted by rearrangement of the video frames, indicating that the discrimination was based on motion cues.

There is also evidence that pigeons can use biological motion cues to categorize stimuli. Using a discriminative autoshaping procedure, Dittrich, Lea, Barrett, and Gurr (1998) found that some pigeons could discriminate video scenes of pigeons pecking from scenes of pigeons walking. On subsequent transfer tests with point-light displays of only the movements, discrimination decreased but remained significantly above chance, suggesting some discrimination based on motion cues alone. Four out of 12 pigeons acquired a discrimination between pecking and walking movements based on only the point-light cues, but none of the birds showed significant transfer to full video displays. Thus, pigeons appear to be capable of discriminating between biologically relevant motion cues, but sensitivity to these cues appears to vary substantially across birds.

Fewer studies have investigated whether motion influences pigeons' encoding or recognition of objects. Cook and Katz (1999) investigated dynamic object recognition in pigeons and provided evidence suggesting that motion may facilitate the perception of 3-D objects. The birds were trained to discriminate between two differently shaped objects (e.g., a cube and a pyramid) that were sometimes presented dynamically (rotating around a particular axis) but on other trials were presented as static objects at randomly selected orientations around an axis. The birds learned a discrimination that remained invariant during a variety of changes, including changes in object size, rate of rotation, and direction of motion. In addition, dynamic presentations supported better performance than did static ones on some transfer tests, including changes in object color and changes to a new axis of rotation.

In contrast to the results of Cook and Katz (1999), Jitsumori and Makino (2004) found little evidence to suggest that motion facilitates pigeons' ability to recognize human faces presented in video images. Pigeons that were trained to discriminate between faces presented in static frontal views showed some generalization to static rotated views of the faces but failed to transfer the discrimination to dynamically rotating faces. Moreover, subsequent training with dynamic views of the faces failed to broaden the generalization to rotated static faces. Overall, the precise role of motion in object recognition in pigeons remains unclear, and further research on this issue is needed.

There is clearer evidence that motion may contribute to object recognition in humans. For example, Stone (1998) trained participants to recognize four target objects rotating in depth and to distinguish them from distractor objects. Reversing the direction of rotation on test trials disrupted the speed and accuracy of recognizing the target objects, suggesting that the spatiotemporal ordering of the views seen during training contributed to the recognition (see also Liu \& Cooper, 2003, who used an old/new recognition task with geometric objects).

Changing an object's learned motion at test has also been shown to impair humans' recognition performance in a four-object identification task across different types of novel objects and training conditions (Vuong \& Tarr, 2006). The findings were especially strong for objects that were difficult to distinguish from each other, whether because their shapes were difficult to decompose, or because they were degraded with visual "dynamic fog" during training. Objects that were easy to decompose did not show any performance decrements when the training and test motions differed. However, in Vuong and Tarr's study, more than one shape was associated with a given motion. Consequently, although motion could serve as a cue to recognition, it was not unique to individual objects.

The present study was designed to extend previous work in both species by providing an initial comparative study on recognition of dynamic objects by pigeons and humans. Of course, many different kinds of motion can be applied to an object to make it dynamic. For example, a rigid object like a rock can be moved by translating it in space along a path or by rotating it around one of its axes; both types of transformation include a direction with respect to some coordinate system (e.g., leftward vs. rightward for translation, or clockwise vs. counterclockwise for rotations in depth). Newell, Wallraven, and Huber (2004) have shown that these two translations differentially affected human categorization of novel dynamic shapes. Alternatively, the object itself could be flexible and move in relation to its parts, as in the case when many animate objects move. Studies of human articulations (e.g., Giese \& Poggio, 2003; Troje, 2002), facial motion (such as expressions; e.g., Knappmeyer, Thornton, \& Bülthoff, 2003; Lander \& Bruce, 2000; Thornton \& Kourtzi, 2002), and even novel deforming shapes (e.g., Chuang, Vuong, Thornton, \& Bülthoff, 2005) have shown that nonrigid motion also facilitates recognition.

Although all of these dynamic properties are interesting to investigate, for our purpose, we focused on the 3-D rotation path of rigid objects. Specifically, both species were trained to discriminate between two rigid objects that were each rotating along the same complex trajectory but in opposite directions. We had three reasons for selecting this kind of motion as a starting point for our comparative work. First, moving different rigid objects in opposite directions along the same path allowed us to equate the number of different views in which each object 
was presented. Second, during test trials, we could present each object in the reverse trajectory from the one that was learned, thus achieving a pure test of the effect of a change in path, holding constant all the views that had been seen during acquisition. Third, rotation paths have been used in previous work with human subjects, and thus we could verify that our results with humans would be consistent with those of previous studies.

In the present study, pigeons and humans learned to identify objects that each had a unique characteristic motion, such that the motion provided a potentially informative cue regarding the identity of the object. Our participants were trained on a go/no-go task to respond to a positive stimulus $(\mathrm{S}+)$ that had a particular object shape and direction of motion, and to withhold responding to a negative stimulus $(\mathrm{S}-$ ) that had a different object shape and an opposite direction of motion. Thus, either object shape or direction of motion could be used to discriminate perfectly between the $\mathrm{S}+$ and $\mathrm{S}-$. Subsequent tests allowed us to determine whether pigeons and humans learned the discrimination on the basis of the shape of the objects, the characteristic object motions, or both properties.

The procedure that we used contrasts with that in previous studies with human participants (e.g., Liu \& Cooper, 2003; Stone, 1998, 1999; Vuong \& Tarr, 2006) in which object shape was implicitly the only reliable cue for correct responses. In these previous studies, either single objects had different rotation trajectories (Stone, 1998, 1999) or several objects shared the same rotation trajectories (e.g., Liu \& Cooper, 2003; Vuong \& Tarr, 2006). Thus, by confounding shape and motion in the present study, we could explore the extent to which humans and pigeons used shape or motion cues within the limitations imposed by the different visual systems, species visual experiences, and training procedures.

We used two types of objects to examine the effect of static object properties on sensitivity to shape and motion in the two species. The objects were identical to those used by Vuong and Tarr $(2004,2006)$. One type consisted of decomposable objects made from distinctive parts (Biederman, 1987; Biederman \& Gerhardstein, 1993), and the other type consisted of nondecomposable, amoeba-like objects. We chose these types of objects because Vuong and Tarr (2004), using the same stimuli, found that humans discriminated the decomposable objects more quickly and accurately than the nondecomposable objects. Similarly, Vuong and Tarr (2006) found that rotation reversal was more detrimental for recognizing the nondecomposable objects than it was for recognizing the decomposable ones.

The difference in discriminability that Vuong and Tarr (2004, 2006) found between decomposable and nondecomposable objects is consistent with extant theories of human object recognition. Specifically, the decomposable objects were constructed of distinctive geons (Biederman, 1987) and they had a defined axis of elongation, whereas the nondecomposable objects were difficult to parse into discriminable parts and they lacked a distinctive axis of elongation (see Vuong \& Tarr, 2004, for further discussion of the objects). Our present interest, however, is not in the perceptual or cognitive differences that might be produced by the qualitative differences between decomposable and nondecomposable objects per se. Indeed, we recognize that decomposable objects (and probably nondecomposable objects) have their own continuum of discriminability (Hayward \& Williams, 2000) and may produce performance differences depending on where along that continuum they are selected. Thus, to investigate the potential interaction between static object properties and sensitivity to motion across species, we selected objects to use in the present study that are known to differ empirically for humans' performance, in order to determine whether pigeons would show similar differences in shape discriminability and sensitivity to motion for these stimuli. Thus, we use the decomposable-nondecomposable terminology in an operational manner as described above, while recognizing that there might be a more theoretically motivated continuum of discriminability underlying this distinction.

In our previous research, we tested pigeons with only decomposable objects (although not the ones used here), and interestingly, the pigeons did not appear to benefit from the presence of a distinctive part in the same way as do humans (Spetch et al., 2001). However, in those studies, we investigated only static images. Pigeons have never been tested with the artificial amoeba-like objects used in the present study, nor have pigeons been used in a comparison between decomposable and nondecomposable objects (whether as static images or as animations). Thus, it is of interest to determine whether pigeons, like humans, would find the amoeba-like objects more difficult to discriminate than the decomposable objects.

For both kinds of objects and both species, we used three kinds of tests to examine the extent to which the direction of motion had been encoded during training. In the first, the directions of motion for the learned stimuli were reversed, providing a conflict between the motion and shape cues. Because one motion and one shape each corresponded to the $\mathrm{S}+$, this test would enable us to see which of the learned cues was the stronger. Second, the learned objects were tested in a new rotation trajectory that differed from the rotation path seen for either the $\mathrm{S}+$ or the $\mathrm{S}-$ object during training. This second test allowed us to assess the degree to which subjects could recognize the objects independently of their characteristic motion. Third, completely new objects were tested in the learned motions to assess whether subjects had encoded the direction of movement independently of the object that had carried the motion.

By allowing shape and motion cues to be perfectly confounded during training, the present study extends previous research on the role of dynamic information in object recognition in two ways. First, because it was possible for motion alone to serve as a cue to object identity, it was possible that we would see decrements in humans' performance with a change in motion even for the objects that were easy to decompose. Second, our study further extends previous work on the role of motion in object recognition by providing a comparison between humans and 
pigeons with the same stimuli and recognition paradigm. Human participants were tested in Experiments 1 and 2, and pigeons were tested in Experiment 3.

\section{EXPERIMENT 1}

\section{Method}

Participants. Twenty-nine volunteers (12 female, 17 male) from the University of Alberta subject pool who were paid on the basis of their task performance served as participants. They were randomly assigned to one of four counterbalanced conditions formed according to which type of object they saw first and which particular object of each pair was the S+. Of these, 8 ( 2 female, 6 male) were eliminated because they did not reach the $80 \%$ correct criterion during the last block of training trials in one or more conditions.

Stimuli and Design. There were two types of stimulus objects and two exemplars of each one. One type of object was made from a large central part and three smaller parts (decomposable objects). The other type was difficult to parse into parts, but still had definite features (nondecomposable objects). Figures 1A and 1B show examples of each, and the Appendix contains links to animations of each stimulus in the training and test motions. The objects were constructed in 3D Studio Max v5.0 (Discrete, Montreal). The 3-D models were then imported into a program written in the C programming language. The program rendered each model with a matte gray surface for each frame of the animation (see Vuong \& Tarr, 2005, for more details).

Animation sequences were made of each object moving through the same rotation trajectory. The sequences were created as follows. First, a virtual camera was placed at a fixed distance away from the centroid of the object. A full $360^{\circ}$ rotation in 3-D space was divided into 128 time steps. At each step, the object was rotated about the $x$-axis, then the $y$-axis, and then the $z$-axis. The magnitude of rotation about each axis was defined by three different functions (see the Appendix). These were controlled by a parameter $t$, which varied from $0^{\circ}$ to $360^{\circ}$ in steps of $\sim 2.8^{\circ}$. This procedure produced 128 frames, each 320 pixels $\times 320$ pixels in size. The animation sequence could be played in ascending frame order for a "clockwise" rotation or in descending order for a "counterclockwise" rotation. The trajectory was periodic, so that the animation sequence could begin at any frame and rotate a complete $360^{\circ}$. All but 1 of the participants received the animations at a rate of 37.5 frames/sec (the movie times were approximately $3.42 \mathrm{sec}$ ). One participant took the test using a different computer monitor and received the movies at $30.0 \mathrm{frames} / \mathrm{sec}$ (movie time $=4.26 \mathrm{sec}$ ). The objects were gray, centered against a yellow background.

Nine participants received the two decomposable objects first, and 12 received the two nondecomposable objects first. During the training phases, approximately half the participants in each objectorder condition responded "go" to object A and "no go" to object $\mathrm{B}$, and the remaining participants received the opposite assignment of objects to response categories. Further, if one object initially moved in a clockwise direction during training, the other moved in a counterclockwise direction. Thus, during training, the shapes of the objects were perfectly confounded with their directions and trajectories of motion. Finally, approximately half of the participants who responded "go" to object A saw it moving in a clockwise direction during training, and the remaining participants saw it moving in a counterclockwise direction; a similar scheme was used for the participants who responded "go" to object B.

In the test phase, there were four types of trial for each object, motion, and response condition seen during training. For example, if a participant had been trained to respond "go" to object A moving clockwise and "no go" to object B moving counterclockwise, then that participant's four types of test trial would be: object A moving clockwise (go-same), object A moving counterclockwise (go-reverse), object B moving counterclockwise (no go-same), and object B moving clockwise (no go-reverse).

Procedure and Apparatus. There were two training phases and one test phase for each type of object, and all the movies on each trial in all phases were presented on a standard 17-in. computer monitor.

When participants arrived at the lab they were told, "your task is to learn to discriminate between two novel objects that are rotating in depth." They were further told that one object would be the correct object, and that it was the one they should respond to; they were to refrain from responding to the other, "wrong," object. They were told that they would at first have to guess, but that soon they would figure out which object was correct and which object was to be ignored.

\section{Training Objects}

\section{Decomposable Objects}
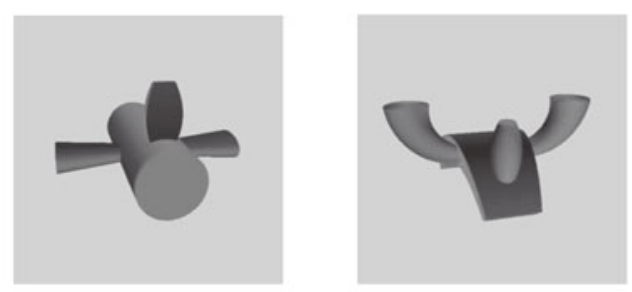

Nondecomposable Objects

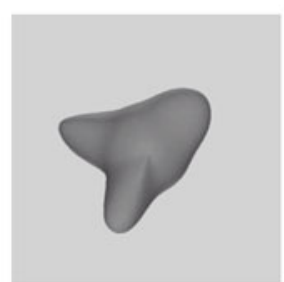

A

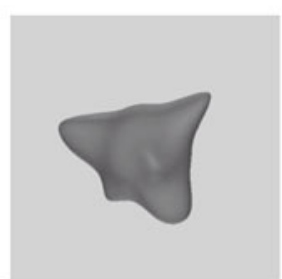

B

\section{Novel Object}
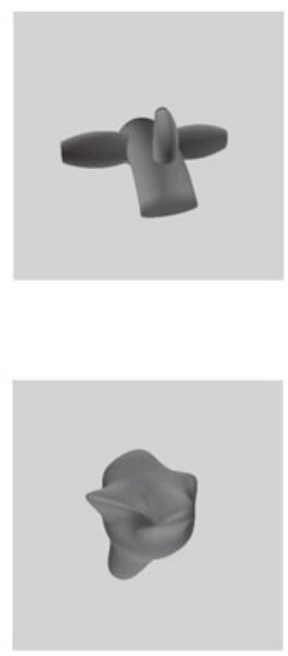

C

Figure 1. Static views of the decomposable and nondecomposable objects used in training (A and B) and for the new-object test (C). 
The participants were next told about the reward scheme for the first training phase: They would receive $2 \phi$ for each correct response ("go" or "no go"), and lose $1 \notin$ for each incorrect response. The participants made their responses by pressing a single button on a box connected to the game port of the computer.

In the first training phase, participants were rewarded for accuracy, not speed. The entire movie was played on each trial, so participants received equal exposure to all views of each object. The starting frame of the movie was randomly determined on a trial-by-trial basis. On each trial, there was a 2.5 -sec blank screen while the movie was being loaded into memory. This 2.5 -sec period served as the intertrial interval (ITI). A fixation point then appeared in the center of the computer monitor for $1 \mathrm{sec}$, and a beep was simultaneously sounded for $200 \mathrm{msec}$. The movie sequence followed immediately upon the offset of the fixation point. After the movie was finished, there was another 200-msec beep to signal that the response period had started. There was a 2-sec window after the movie was finished, during which the participant could respond once if it was a go trial and had to refrain from responding if it was a no-go trial. Feedback was given on the computer screen with either the word "Correct" or the word "Wrong" presented after the 2-sec response window. There were 40 trials during this phase ( 20 each for go and no go), and at the end of these, the participants were told how much they had earned thus far.

During the second training phase, we introduced a reward scheme designed to encourage both accuracy and speed. Accurate go and no-go responses still received a $2 \notin$ reward; in addition, go responses that were faster than $1 \mathrm{sec}$ earned an additional $3 \phi$ (5ф total). Incorrect responses again caused $1 \varnothing$ to be subtracted from the total. The participants were told that they should respond on go trials as soon as they knew the answer, but they were not told the exact time that would earn them the $5 \phi$ reward. The entire movie sequence was played on both go and no-go trials. The participants could respond until $1 \mathrm{sec}$ after the end of the movie before the feedback screen appeared. The feedback message for an accurate go response greater than $1 \mathrm{sec}$ and an accurate no-go response was "Correct, you earn $2 \notin$ "; the message for accurate go responses that were made in less than $1 \mathrm{sec}$ was "Correct, you earn $5 \phi$ "; and the message for incorrect responses was "Wrong, you lose $1 \notin$. ." The participants were again told the total they had earned at the end of the training block. There were 40 trials in the second phase (20 each for go and no go).

During the test phase, participants were told that the procedure and reward scheme were identical to those in the previous practice phase ( $2 \phi$ for correct go and no-go responses, and $5 \phi$ if the go responses were fast), except that they would not be receiving feedback after each trial. They were not told anything about the novel motions. There were 80 trials during the test phase: 20 each for go-same motion, go-reverse motion, no go-same motion, and no go-reverse motion. These test trials were presented in a randomized order within blocks of four trials. Correct responses for determining the accumulation of rewards during testing were based on object shape, but the participants were not told this and did not receive feedback about accumulated points until the end of testing.

\section{Results}

Training trials. To compare ease of learning about the decomposable and nondecomposable objects, we scored the number of correct responses (across go and no-go trials) for the first and second training blocks, for which participants were under accuracy and accuracy plus speed instructions, respectively. The means during the first block of training for decomposable and nondecomposable objects were $87.3 \%$ and $76.9 \%$, respectively $[t(20)=2.74$, $\left.S D_{\text {diff }}=.175, p=.013\right]$. The means during the second block of training for decomposable and nondecomposable objects were $98.5 \%$ and $96.4 \%$, respectively $[t(20)=$ 2.74, $\left.S D_{\text {diff }}=.175, p=.033\right]$. Thus, participants were more accurate on the decomposable objects than they were on the nondecomposable objects from the first block of training trials.

Test trials. To compare the results that we obtained with the present go/no-go procedure with those obtained by Vuong and Tarr (2006) with a four-choice identification procedure, we first scored the data for overall accuracy and analyzed them in an object type (decomposable, nondecomposable) $\times$ motion type (same, reverse) repeated measures ANOVA. Note that accuracy was scored on the basis of shape. Both main effects were reliable $\left[F(1,20)=23.94, M S_{\mathrm{e}}=0.001, \eta_{\mathrm{p}}^{2}=.545\right.$, for object type; and $F(1,20)=5.05, M S_{\mathrm{e}}=0.143, \eta_{\mathrm{p}}^{2}=.201$, for motion type]. When the training and test motions were the same, responses were more accurate than when they were different $(99.0 \%$ and $80.0 \%$, respectively), and participants were slightly less accurate with the nondecomposable objects than with the decomposable objects $(88.0 \%$ and $90.6 \%$, respectively). Most important, the effect of reversing the motion had the same detrimental effect on accuracy for both stimulus types (see Table 1) $[F(1,20)=$ $5.21, M S_{\mathrm{e}}=0.141, \eta_{\mathrm{p}}^{2}=.206$, for the decomposable objects; and $F(1,20)=4.84, M S_{\mathrm{e}}=0.146, \eta_{\mathrm{p}}^{2}=.195$, for the nondecomposable objects]. Thus, we established that the go/no-go procedure used in the present study replicated all of the basic phenomena observed by Vuong and Tarr (2006; see also Stone, 1998). ${ }^{1}$

We next scored the data according to the proportion of go responses made in each object type (decomposable or nondecomposable), trial type (go, no go), and motion type (same or reverse) condition (out of a total of 20 trials per condition) and analyzed the results in a repeated measures ANOVA. All of the main effects were significant, as were

Table 1

Proportion Correct on Same- and Reverse-Motion Trials As a Function of Object Type (Decomposable, Nondecomposable) in Experiments 1 and 2 (Human Participants)

\begin{tabular}{lccccc}
\hline & \multicolumn{2}{c}{ Decomposable Objects } & & \multicolumn{2}{c}{ Nondecomposable Objects } \\
\cline { 2 - 3 } \cline { 5 - 6 } & Same Motion & Reverse Motion & & Same Motion & Reverse Motion \\
\hline Experiment 1 & .998 & .814 & & .973 & .786 \\
Experiment 2 & .976 & .863 & & .948 & .750
\end{tabular}

Note-In Experiment 1, the 95\% confidence interval on the four means for this repeated measures ANOVA was .014 (Loftus \& Masson, 1994). In Experiment 2, the 95\% confidence interval on the four means for this repeated measures ANOVA was .048 (Loftus \& Masson, 1994) 
Table 2

ANOVA Results for the Proportion of Go Responses by Humans (Experiments 1 and 2) and Pigeons (Experiment 3) As a Function of Object Type (Decomposable, Nondecomposable), Trial Iype (Go, No Go), and Motion Iype (Same, Reverse)

\begin{tabular}{|c|c|c|c|c|c|c|c|c|c|}
\hline \multirow[b]{2}{*}{ Source } & \multicolumn{3}{|c|}{$\begin{array}{c}\text { Experiment } 1 \\
\left(d f_{\mathrm{s}}=1,20\right)\end{array}$} & \multicolumn{3}{|c|}{$\begin{array}{c}\text { Experiment } 2 \\
\left(d f_{\mathrm{s}}=1,23\right)\end{array}$} & \multicolumn{3}{|c|}{$\begin{array}{c}\text { Experiment } 3 \\
\left(d f_{\mathrm{s}}=1,8\right)\end{array}$} \\
\hline & $F$ & $M S_{\mathrm{e}}$ & $\eta_{\mathrm{p}}^{2}$ & $F$ & $M S_{\mathrm{e}}$ & $\eta_{\mathrm{p}}^{2}$ & $F$ & $M S_{\mathrm{e}}$ & $\eta_{\mathrm{p}}^{2}$ \\
\hline Object type (O) & $12.87^{\mathrm{a}}$ & .0014 & .391 & $<1$ & & & $6.34^{\mathrm{a}}$ & .022 & .475 \\
\hline Trial type $(\mathrm{T})$ & $88.67^{\mathrm{a}}$ & .2920 & .816 & $106.44^{\mathrm{a}}$ & .266 & .822 & $109.03^{\mathrm{a}}$ & .023 & .940 \\
\hline Motion type (M) & $5.56^{\mathrm{a}}$ & .0005 & .218 & $<1$ & & & $3.76^{\mathrm{a}}$ & .021 & .350 \\
\hline $\mathrm{O} \times \mathrm{T}$ & $23.94^{\mathrm{a}}$ & .0013 & .545 & $9.31^{\mathrm{a}}$ & .025 & .288 & $166.69^{a}$ & .003 & .960 \\
\hline $\mathrm{O} \times \mathrm{M}$ & $3.65^{\mathrm{b}}$ & .0003 & .155 & $<1$ & & & $1.54^{\mathrm{c}}$ & .019 & .180 \\
\hline $\mathrm{T} \times \mathrm{M}$ & $5.04^{\mathrm{a}}$ & .2853 & .201 & $5.61^{\mathrm{a}}$ & .207 & .196 & $205.18^{\mathrm{a}}$ & .008 & .970 \\
\hline $\mathrm{O} \times \mathrm{T} \times \mathrm{M}$ & $<1$ & $<1$ & & $3.35^{b}$ & .026 & .126 & $15.97^{\mathrm{a}}$ & .027 & .695 \\
\hline
\end{tabular}

the two-way interactions between object type and trial type and between trial type and motion type; the $F$ ratios and effect sizes are shown in Table 2, and the means are shown in Figure 2.

The results of four planned contrasts provided support for the hypothesis that the reverse motion condition would be more difficult on go and no-go trials for both decomposable and nondecomposable objects. The mean proportions of go responses on go trials for the same- and reverse-motion tests were 1.00 and .82 , respectively, for the decomposable objects and .99 and .82 for the nondecomposable objects; both differences were significant $\left[F(1,20)=4.69, M S_{\mathrm{e}}=\right.$ $0.147, \eta_{\mathrm{p}}^{2}=.190$, and $F(1,20)=4.37, M S_{\mathrm{e}}=0.145, \eta_{\mathrm{p}}^{2}=$

\section{Humans: Experiment 1 Decomposable}

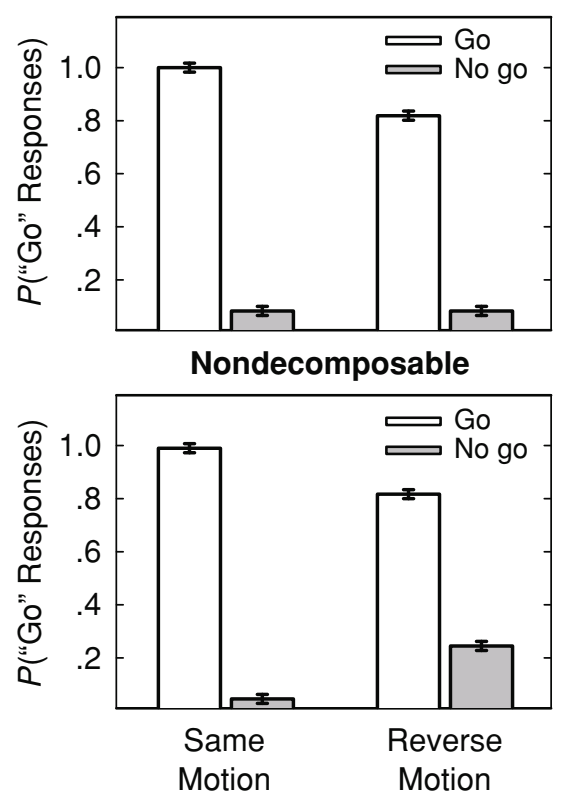

Figure 2. The proportion of go responses for human participants in Experiment 1 as a function of object type (decomposable, nondecomposable), trial type (go, no go), and motion type (same, reverse). Error bars are the $\mathbf{9 5 \%}$ confidence intervals for this repeated measures design (Loftus \& Masson, 1994).
.179 , for the decomposable and nondecomposable objects, respectively]. Similarly, the mean proportions of go responses on no-go trials for the same- and reverse-motion tests were .01 and .19 , respectively, for the decomposable objects and .05 and .25 for the nondecomposable objects; both differences were significant $\left[F(1,20)=4.98, M S_{\mathrm{e}}=\right.$ $0.145, \eta_{\mathrm{p}}^{2}=.199$, and $F(1,20)=6.11, M S_{\mathrm{e}}=0.137, \eta_{\mathrm{p}}^{2}=$ .233 , for the decomposable and nondecomposable objects, respectively].

There was a possibility that the results on the test trials reflected an eventual loss of stimulus control due to the cessation of explicit reinforcement. To test this idea, we reanalyzed only the last block of trials in each condition for both the percentage correct and proportion of go responses (there were 20 and 10 trials/condition, respectively). All the effects reported above were significant on the last block of trials for both measures, and they were of a magnitude virtually identical to that for the complete data set. For example, the mean proportions of go responses for the last 10 go trials in the same- and reverse-motion conditions were 1.00 and .81 for the decomposable objects and .99 and .81 for the nondecomposable objects, respectively. Similarly, the mean proportions of go responses on the last 10 no-go trials for same- and reverse-motion conditions were 0 and .19 for the decomposable objects and .05 and .24 for the nondecomposable objects. Thus, we do not think that the detrimental effect of reversing the trajectories of the objects was due to a loss of stimulus control.

To summarize, for both decomposable and nondecomposable objects, reversing the motion reduced correct responses (based on shape alone) on both go and no-go trials. On go test trials, reversing the motion diminished the proportion of go responses by $17.7 \%$ relative to the proportion of go responses on same-motion trials. On no-go test trials, reversing the motion increased the proportion of go responses relative to the proportion of go responses on same-motion trials by $19.3 \%$.

\section{EXPERIMENT 2}

The reverse-motion tests of Experiment 1 provided a conflict between information provided by the shape of each learned object and information provided by the char- 
acteristic motion of each object. For both object types, participants continued to respond with above chance accuracy on the basis of object shape, but the reduction in accuracy on reverse-motion tests relative to same-motion tests indicates that motion played some role in the identification of the objects. Experiment 2 was designed to extend this finding in two ways. First, the tests were replicated but with instructions that were designed to be unbiased toward the object feature (shape or motion) that should be used to solve the discrimination. Second, additional tests were conducted to assess control by shape or motion cues alone. The latter tests entailed presenting new object shapes in the learned motions or new trajectories for the learned object shapes. That is, the new trajectories were not simply a reversal of the frame sequences of the learned trajectories.

\section{Method}

Participants. Thirty-one volunteers ( 22 female, 9 male) from the University of Alberta subject pool who were paid on the basis of their task performance served as participants. They were randomly assigned to one of four counterbalanced conditions formed according to which type of object they saw first and which particular object of each pair was the $\mathrm{S}+$. Of these, 7 ( 5 female, 2 male) were eliminated because they did not reach the $80 \%$ correct criterion during the last block of training trials in one or more conditions.

Stimuli and Design. Both of the decomposable and nondecomposable objects and animation trajectories from Experiment 1 were used as training stimuli, and we made one additional exemplar of each object type (see Figure 1C) to use as a new-object test stimulus. The new object was presented in the identical (clockwise or counterclockwise) training trajectories as were the original training stimuli. We also created an entirely new trajectory in which to display the original training objects. This trajectory was created as described above in Experiment 1, but with the rotation control parameter $t$ varying from $360^{\circ}$ to $0^{\circ}$ in steps of $\sim 2.8^{\circ}$. Therefore, the new trajectory had the same complexity as that of the old trajectory.

The training scheme was similar in most respects to that used in Experiment 1. Fourteen participants were trained with decomposable objects first, and 10 were trained with nondecomposable objects first. For half of the participants in each object order group, object A was the S+ and moved in a clockwise trajectory and object $\mathrm{B}$ was the $\mathrm{S}-$ and moved in the counterclockwise trajectory. The remaining participants received the reverse assignment of objects to directions. Nineteen participants received the animations at $37.5 \mathrm{frames} / \mathrm{sec}$ (the movie times were approximately $3.42 \mathrm{sec}$ ). Five participants received their tests on different monitors; 3 of these participants received the animations at 29.8 frames $/ \mathrm{sec}$ (movie time $=$ $4.30 \mathrm{sec}$ ), and the remaining 2 participants received the stimuli at $25.0 \mathrm{frames} / \mathrm{sec}$ (movie time $=5.12 \mathrm{sec}$ ).

There were eight types of test trials for both decomposable and nondecomposable objects. Four of the test trials were an exact replication of the same- and reverse-motion tests of Experiment 1. These trials were analyzed separately to provide an internal replication of the results of Experiment 1.

There were also four new types of test trials (see the new motion and new object animations in the Appendix). Two of these were new motion tests in which the trained go and no-go objects were presented using the altogether new stimulus trajectory. If participants responded entirely on the basis of shape, then the old S+ object should still evoke a go response and the old $\mathrm{S}-$ object should still evoke a no-go response. But if motion cues were encoded together with the shape cues, then the new trajectory might disrupt the discrimination between the $\mathrm{S}+$ and $\mathrm{S}-$ object shapes.

The remaining new trials presented a completely new object (of the same type as the old objects - decomposable or nondecompos- able) in either the old $\mathrm{S}+$ trajectory or the old $\mathrm{S}-$ trajectory. On these trials, the motion type (trajectory plus clockwise or counterclockwise direction) was identical to that used in training, but the object shape was different. If the specific motion trajectories were encoded somewhat independently of the training shapes, then participants might respond according to the training trajectories even though the test shape was novel; that is, they might respond "go" to the old S+ trajectory and refrain from responding when the new object was moving in the old S- trajectory.

Procedure. The training procedure was identical to that in Experiment 1 , except that the instructions were rewritten to make both shape and motion plausible bases for a correct response. In particular, references to "the correct object" were replaced with phrases such as "the correct movie" or "animation sequence." For example, at the beginning of the instructions, participants were told, "In this phase, you will learn to discriminate between two stimulus displays that are shown as animated movies. Each movie shows one of two novel objects rotating along one of two particular paths. One movie will be 'correct' and one will be 'wrong." In this way, we hoped to eliminate any bias in the instructions that might have discouraged the use of motion cues. Otherwise, the two training phases were identical to the training in Experiment 1.

The payoff scheme was altered slightly from that in Experiment 1. During the first training phase, participants received $1 \phi$ for each correct go or no-go response and $1 \notin$ was subtracted from their score for each incorrect response. During the second training phase, they received $1 \notin$ for each correct go response that was longer than $1 \mathrm{sec}$, $1 \phi$ for each correct no-go response, and $3 \phi$ for each correct go response that was faster than $1 \mathrm{sec}$. One cent was subtracted from their score for each incorrect response. They received feedback after each trial, in the form "Correct, you earn 1 $\phi$," or "Correct, you earn $3 \phi$," or "Wrong, you lose $1 \phi$," and the total amount that they had earned at the end of each block appeared on the computer screen.

The instructions during the test phase were modified to remain neutral with respect to whether correct responding was to be rewarded on the basis of shape or of motion. Specifically, participants were told that "Some of the animations you see during this phase will differ in some way from the ones you saw previously. You should try to decide whether to respond Go or No Go based on what you think is most appropriate, given your previous feedback." Correct responses were based on shape for same-motion, reversemotion, and new-motion trials, but for new-object trials, participants were rewarded for any response (any fast go responses earned $3 \phi$ ). However, the participants were not told this, and they did not receive feedback, so this assignment of rewards to choices could not affect their performance.

During the test phase, participants received 20 of each type of test trial, presented in a randomized order within blocks of 8 trials, for a total of 160 trials. The procedure was identical to that in Experiment 1.

\section{Results}

Training trials. We again scored the number of correct responses (based on shape, across go and no-go trials) for the first and second training blocks, for which participants were under accuracy and accuracy plus speed instructions, respectively. The means for decomposable and nondecomposable objects did not differ during the first block of training $(74.0 \%$ and $78.1 \%$, respectively, $p=.231$ ), but mean accuracy was significantly higher for the decomposable objects $(97.5 \%)$ than for the nondecomposable objects $(95.4 \%)$ during the second block of training trials $\left[t(23)=2.69, S D_{\text {diff }}=.040, p=.015\right]$. Thus, the difference in accuracy between decomposable and nondecomposable objects took longer to develop in this experiment than it did in Experiment 1. 
Test trials. For this experiment, we analyzed the sameand reverse-motion tests in a separate repeated measures ANOVA from the new-object and new-motion tests. We did this primarily because the same- and reverse-motion tests provided an exact replication of Experiment 1, and these conditions can be meaningfully scored for accuracy. Thus, we could determine whether adding the new-object and new-motion tests adversely affected overall performance in the replication conditions. The new-object and new-motion tests themselves provided a means of disentangling the extent to which each kind of originally encoded information (old motion and old shape) affected the tendency to respond or to inhibit responding.

Same- and reverse-motion tests. We again first scored the data for overall accuracy on same- and reversemotion trials for each type of object (based on shape) and analyzed them in an object type (decomposable, nondecomposable) $\times$ motion type (same, reverse) repeated measures ANOVA. As in Experiment 1, both main effects were reliable $\left[F(1,23)=9.31, M S_{\mathrm{e}}=0.013, \eta_{\mathrm{p}}^{2}=.288\right.$, for object type; and $F(1,23)=5.61, M S_{\mathrm{e}}=0.103, \eta_{\mathrm{p}}^{2}=$ .196 , for motion type]. Participants were more accurate in responding to decomposable objects than to nondecomposable objects ( $92.0 \%$ and $84.9 \%$, respectively), and they were more accurate when the study and test directions were the same than when they were reversed $(96.2 \%$ and $80.7 \%$, respectively). Further, post hoc tests showed that performance was significantly more accurate with decomposable objects than with nondecomposable objects on both same-motion trials $\left[F(1,23)=9.62, M S_{\mathrm{e}}=\right.$ $\left.0.002, \eta_{\mathrm{p}}^{2}=.295\right]$ and reverse-motion trials $[F(1,23)=$ $\left.6.20, M S_{\mathrm{e}}=0.049, \eta_{\mathrm{p}}^{2}=.212\right]($ see Table 1$)$.

We next analyzed the proportion of go responses made in each of the four replication conditions for each object type. The $F$ ratios and effect sizes are shown in Table 2, and the means are shown in the two leftmost pairs of bars in the top and bottom panels of Figure 3. Some of the differences were not as large as they were in Experiment 1, but the overall pattern of responding was the same. In particular, the results of the planned comparisons showed that for the decomposable objects, the difference in proportion of go responses to same- and reverse-motion trials only approached significance, both for trials when go was the correct answer ( $M=1.00$ vs. 0.90 , respectively) $[F(1,23)=$ 3.14, $\left.M S_{\mathrm{e}}=0.083, \eta_{\mathrm{p}}^{2}=.120, p=.089\right]$ and for trials when no go was correct ( $M=0.05$ vs. 0.17 , respectively) $\left[F(1,23)=3.53, M S_{\mathrm{e}}=0.103, \eta_{\mathrm{p}}^{2}=.133, p=.073\right]$. In contrast, for the nondecomposable objects, the difference between same- and reverse-motion trials was significant when go was the correct response ( $M=0.98$ vs. 0.78 , respectively) $\left[F(1,23)=6.90, M S_{\mathrm{e}}=0.139, \eta_{\mathrm{p}}^{2}=.231\right]$ and when no go was correct ( $M=0.08$ vs. 0.28 , respectively) $\left[F(1,23)=6.12, M S_{\mathrm{e}}=0.150, \eta_{\mathrm{p}}^{2}=.210\right]$.

Across decomposable and nondecomposable objects in Experiment 2, reversing the motion on go trials diminished the proportion of go responses by $15.2 \%$, relative to the proportion of go responses on same-motion trials. On nogo test trials, reversing the motion increased the proportion of go responses, relative to the proportion of go responses

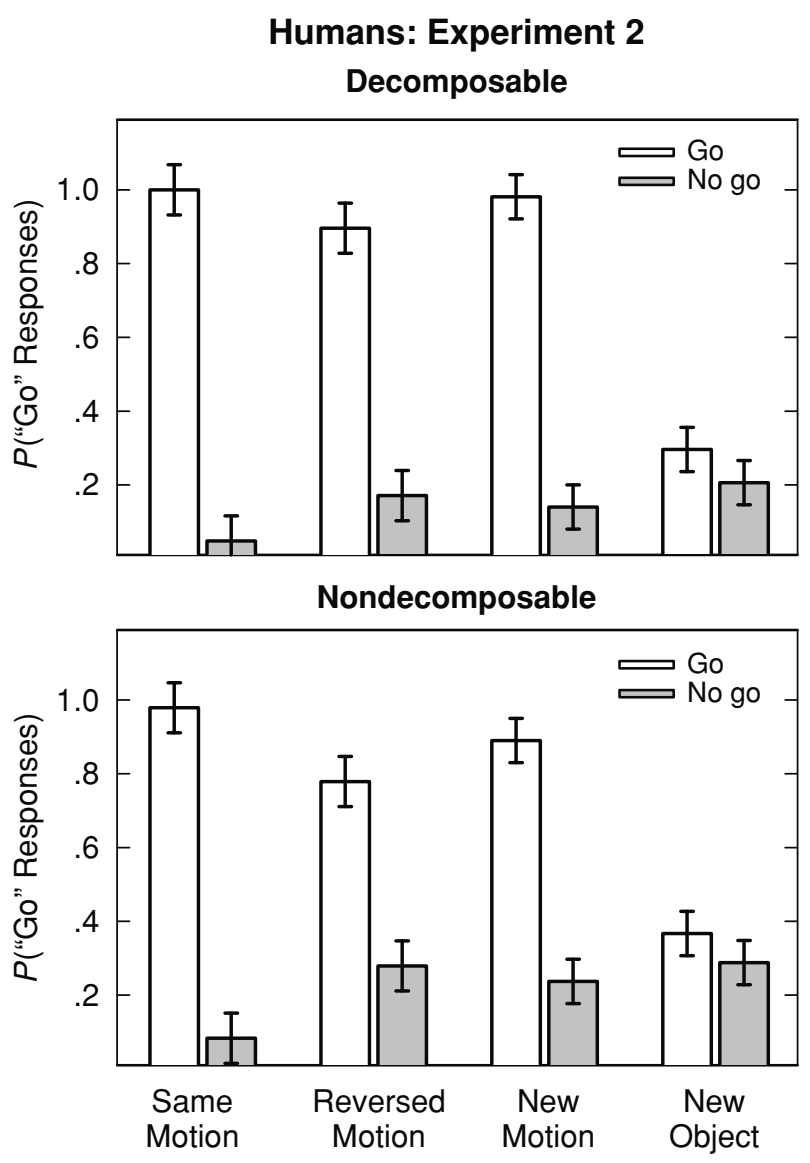

Figure 3. The proportion of go responses for human participants in Experiment 2 as a function of object type (decomposable, nondecomposable), trial type (go, no go), and type of test condition. On same-motion tests, both the shape and motion were identical to training. On reverse-motion tests, only the motion was reversed from training. For the new-motion test, the trained objects were presented in a new motion. For the new-object tests, the trained motions were presented in a new shape. Error bars are the $\mathbf{9 5 \%}$ confidence intervals for a repeated measures design (Loftus \& Masson, 1994), computed separately from the ANOVAs for the same- and reverse-motion conditions and for the new-motion and new-object conditions.

on same-motion trials, by $15.8 \%$. Thus, we replicated the finding that participants encoded the motion of the objects during the training trials, and this affected their responses during testing when the motion was reversed. Furthermore, these results were not caused by a loss of stimulus control. As in Experiment 1, we repeated the analyses for just the last block of trials for each condition (20 trials/condition for the percentage correct data, and 10 trials/condition for the proportion of go responses). All of the effects remained significant on the last block of trials, and again, their magnitude was virtually identical to that for the complete data set.

New-object and new-motion tests. The more important data for this experiment were the go and no-go test trials involving either a completely new object or a completely new motion trajectory. We analyzed the proportion of go responses to the new conditions in an object 
type (decomposable, nondecomposable) $\times$ trial type (go, no go) $\times$ change type (new object, new motion) repeated measures ANOVA. The results are shown in Table 3, and the means are shown in the two rightmost pairs of bars in the top and bottom panels of Figure 3.

Planned comparisons showed that when the old objects were seen in a brand new trajectory, the proportion of go responses was significantly higher on go than on nogo trials for both the decomposable objects $[F(1,23)=$ $\left.149.06, M S_{\mathrm{e}}=0.114, \eta_{\mathrm{p}}^{2}=.866\right]$ and the nondecomposable objects $\left[F(1,23)=58.72, M S_{\mathrm{e}}=0.174, \eta_{\mathrm{p}}^{2}=.719\right]$. However, when the brand new object was seen in the old trajectories, the proportion of go responses was not significantly different from the proportion of no-go responses for either decomposable objects $\left[F(1,23)=1.38, M S_{\mathrm{e}}=\right.$ $\left.0.139, \eta_{\mathrm{p}}^{2}=.057, p=.252\right]$ or nondecomposable objects $\left[F(1,23)=1.73, M S_{\mathrm{e}}=0.231, \eta_{\mathrm{p}}^{2}=.070, p=.201\right]$.

We repeated all the analyses reported above on the last 10 trials of each object type for each trial type and each change type condition. All of the findings reported as significant across all the trials were significant when just the last 10 trials per condition were analyzed, and again, they were of virtually identical magnitude. Thus, the results for humans do not appear to reflect a loss of stimulus control.

In summary, the data indicate that humans are sensitive to motion cues when they learn to discriminate objects, especially objects that are difficult to parse into distinct features. They tend to make fewer go responses to old $\mathrm{S}+$ objects shown in new motions (either the reverse of the training motion or a completely new motion) than when the old S+ objects are shown in the same motion that they were trained in. However, the motion cues did not appear to be encoded independently of the object cues, because participants did not respond differentially to the old $\mathrm{S}+$ and $\mathrm{S}-$ trajectories when they were embodied in an entirely new object context. Thus, while performance was affected by motion cues, it was not to the exclusion of shape information.

\section{EXPERIMENT 3}

In this experiment, we trained pigeons in a go/no-go task with the same dynamic objects used for humans in

Table 3

ANOVA Results for the Proportion of Go Responses by Humans (Experiment 2) and Pigeons (Experiment 3) As a Function of Object Type (Decomposable, Nondecomposable), Trial Type (Go, No Go), and Change Type (New Object, New Motion)

\begin{tabular}{lcccccccc}
\hline & \multicolumn{3}{c}{$\begin{array}{c}\text { Experiment } 2 \\
(d f \mathrm{~s}=1,23)\end{array}$} & & \multicolumn{3}{c}{$\begin{array}{c}\text { Experiment } 3 \\
(d f \mathrm{~s}=1,8)\end{array}$} \\
\cline { 2 - 3 } \cline { 6 - 8 } \multicolumn{1}{c}{ Source } & $F$ & $M S_{\mathrm{e}}$ & $\eta_{\mathrm{p}}^{2}$ & & $F$ & $M S_{\mathrm{e}}$ & $\eta_{\mathrm{p}}^{2}$ \\
\hline Object type (O) & $<1$ & $<1$ & & & $3.39^{\mathrm{b}}$ & .040 & .327 \\
Trial type (T) & $80.51^{\mathrm{a}}$ & .103 & .778 & & $8.86^{\mathrm{a}}$ & .024 & .559 \\
Change type (C) & $18.65^{\mathrm{a}}$ & .192 & .449 & & $52.03^{\mathrm{a}}$ & .017 & .881 \\
$\mathrm{O} \times \mathrm{T}$ & $12.58^{\mathrm{a}}$ & .010 & .354 & & $<1$ & & \\
$\mathrm{O} \times \mathrm{C}$ & $<1$ & & & & $6.35^{\mathrm{a}}$ & .022 & .478 \\
$\mathrm{~T} \times \mathrm{C}$ & $32.28^{\mathrm{a}}$ & .163 & .584 & & $<1$ & & \\
$\mathrm{O} \times \mathrm{T} \times \mathrm{C}$ & $4.75^{\mathrm{a}}$ & .020 & .171 & & $20.56^{\mathrm{a}}$ & .010 & .746 \\
\hline${ }^{\mathrm{a}} p<.05 . \quad{ }^{\mathrm{b}} p=.11$. & & & & & &
\end{tabular}

Experiments 1 and 2. The pigeons were then given the same-motion, reversed-motion, new-object, and newtrajectory tests.

\section{Method}

Subjects. The subjects were 8 adult pigeons (Columba livia). All had previous experience in tasks conducted with computer screens, including a previous study on simultaneous discriminations of static objects. None had previously been trained with dynamic stimuli. The birds were maintained at approximately $85 \%$ of their free-feeding weights by pigeon pellets obtained during experimental sessions and supplemental feedings in the home cages. The birds were housed in large individual cages under a 12:12-h light:dark cycle (with light onset at 6:00 a.m.). Grit and water were freely available in the home cages.

Stimuli and Design. The stimuli were identical to those used for humans and the design was similar to that of Experiment 2, except that training and testing entailed multiple sessions. The object that served as the $\mathrm{S}+$ object was counterbalanced across birds. The pigeons were trained to criterion and then given same- and reversemotion tests with each object type in counterbalanced order across birds. When all of these tests were complete, the pigeons received retraining and were then given new-object and new-motion tests with both decomposable and nondecomposable objects, again in counterbalanced order.

Apparatus. The pigeons were tested in a large custom-built operant chamber, $44 \mathrm{~cm}$ high, $32 \mathrm{~cm}$ deep, and $74 \mathrm{~cm}$ wide (inside dimensions), equipped with a 15 -in. color monitor, a Carroll Touch infrared touch frame that recorded the $x$ - and $y$-coordinates of the pigeons' pecks, and two solenoid-type bird feeders, one on each side of the monitor. Lamps located within each feeder illuminated feeder presentations, and photocells measured the duration of head entries into the hoppers to limit feeding durations to $2 \mathrm{sec}$ per food presentation. The chambers were connected to microcomputers located in an adjacent room. These computers controlled all of the experimental contingencies and recorded the responses.

Training. The pigeons received one session per day, 5 or 6 days per week. Each trial began with the presentation of either the S+ animation or the $\mathrm{S}-$ animation. On go trials, the stimulus display lasted for a minimum of one full rotation of the object $(4,260 \mathrm{msec})$. If a peck was recorded in the area containing the stimulus $(14 \mathrm{~cm}$ wide $\times 12 \mathrm{~cm}$ high), the display ended immediately following the full rotation. If no peck was recorded, the rotating stimulus stayed on until a peck was recorded or for a maximum of $1 \mathrm{~min}$. All go trials in which a peck occurred ended with $2 \mathrm{sec}$ of food reinforcement, followed by a 5 -sec ITI, during which the stimulus display was off. Go trials without a peck were followed directly by the ITI. On no-go trials, the stimulus display remained on only for a full rotation. If the pigeon pecked during the no-go display, the trial ended with a 10 -sec time-out period in which the stimulus display was red, followed by the ITI. If the pigeon did not peck, the no-go trial was followed directly by the ITI. During training sessions, go and no-go trials occurred equally often in a random order within blocks of eight trials and sessions lasted for $45 \mathrm{~min}$. The birds were trained until they reached a criterion of five consecutive sessions in which discrimination ratios (calculated as pecks on go trials divided by pecks on go and no-go trials) reached or exceeded .8. For all data analyses, only the pecks recorded during the first full motion were used on go trials, so that response opportunity was equated for go and no-go trials.

Same- and reverse-motion tests. Each testing session contained 80 trials, 40 of which were training trials that provided reinforcement and time-outs, and 40 of which were test trials in which no reinforcement or time-outs were presented. The order of training and test trials was randomized within each block of 8 trials. Half of the test trials were same-motion trials in which the object and the motion were identical to those in training. The remaining test trials were reverse-motion trials in which the direction of motion was reversed 
for each object, in such a way that the $\mathrm{S}+$ object was presented in the $\mathrm{S}-$ motion and the $\mathrm{S}-$ object was presented in the $\mathrm{S}+$ motion. On all test trials, the object remained on for one full rotation, during which pecks were recorded. Each pigeon received 10 test sessions.

Following testing, each bird was then trained and tested with the other object type (decomposable or nondecomposable) using the identical procedures.

New-object and new-motion tests. Following the last set of same- and reverse-motion tests, the birds were put back on training (half with the most recently tested object type, and half with their first object type), until their discrimination ratio was $80 \%$ or higher for 2 consecutive sessions. They then received 5 sessions of testing that were similar to the previous testing sessions. Each testing session contained 84 trials, half of which were training trials that provided reinforcement and time-outs, and half of which provided no reinforcement or time-outs. Twelve of these nonreinforced trials presented the training stimuli (S+ object in $\mathrm{S}+$ trajectory, and $\mathrm{S}-$ object in $\mathrm{S}-$ trajectory), 12 were new-motion tests in which the $\mathrm{S}+$ and $\mathrm{S}-$ training objects were presented in a novel trajectory, and 12 were new-object tests in which a new object was rotated in the $\mathrm{S}+$ and $\mathrm{S}-$ trajectories. The order of training and test trials was randomized within blocks of 12 trials. The new objects and motions were identical to those used in Experiment 2 and thus assessed whether the pigeons could discriminate on the basis of shape alone, or motion alone, respectively. After testing with one object type, the birds were trained and tested with the other object type using the same procedure.

\section{Results}

Training trials. The birds were trained to criterion separately for each object type. Although the trials to criterion were quite variable across birds (the range was from 7 to 96 trials), the average number of trials to reach the training criterion with the decomposable objects was significantly less than the average for the nondecomposable stimuli (15.1 vs. 43.1 , respectively) $[t(7)=2.38, p<.05]$.

Test trials: Same- and reverse-motion tests. We analyzed the results on the test trials for the birds in the same way as for the humans in Experiment 2, using one repeated measures ANOVA for the same- and reverse-motion trials and a second for the new-motion and new-object trials. The mean proportion of go responses on the same- and reverse-motion tests are shown in the two leftmost pairs of bars in the top and bottom panels of Figure 4. For both types of objects, the birds showed excellent discrimination on the same-motion tests. The tendency to respond more on go trials than on no-go trials decreased slightly for the decomposable objects and dramatically for the nondecomposable objects when the motion was reversed. The results of a repeated measures ANOVA with object type (decomposable, nondecomposable), trial type (go, no go), and motion type (same and reversed) as factors are given in Table 2, along with the effect sizes. The significant three-way interaction confirmed the observation that for pigeons, the discrimination was affected by the motion change more for the nondecomposable objects than for the decomposable objects.

Planned comparisons on the reverse-motion tests revealed that the pigeons responded significantly more on go trials than on no-go trials for the decomposable objects $\left[F(1,7)=36.57, M S_{\mathrm{e}}=0.041, \eta_{\mathrm{p}}^{2}=.839\right]$, but responded significantly less on go trials than on no-go trials for the nondecomposable objects $\left[F(1,7)=16.98, M S_{\mathrm{e}}=0.034\right.$,

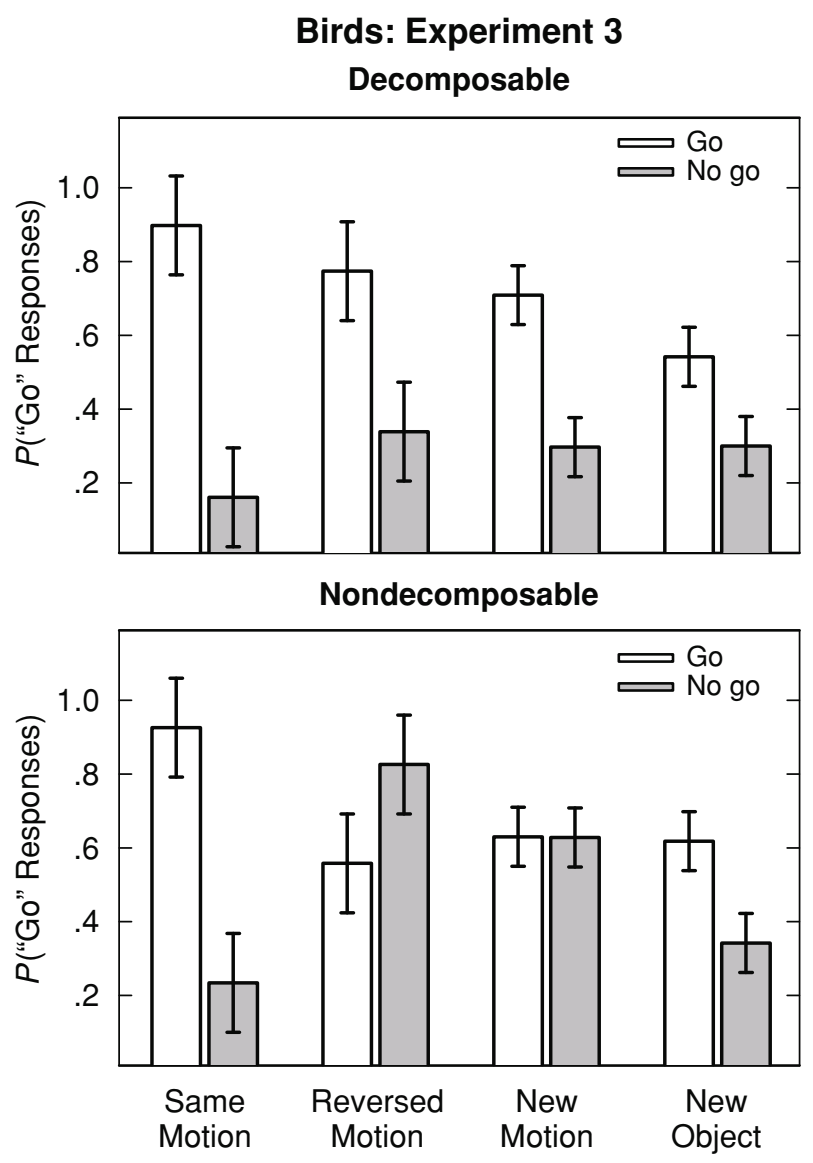

Figure 4: Proportion of go responses for pigeon subjects in Experiment 3 as a function of object type (decomposable, nondecomposable), trial type (go, no go), and type of test condition. On same-motion tests, both the shape and motion were identical to training. On reverse-motion tests, only the motion was reversed from training. For the new-motion test, the trained objects were presented in a new motion. For the new-object tests, the trained motions were presented in a new shape. Error bars are the $95 \%$ confidence intervals for a repeated measures design (Loftus \& Masson, 1994), computed separately from the ANOVAs for the same- and reverse-motion conditions and for the new-motion and new-object conditions.

$\left.\eta_{\mathrm{p}}^{2}=.708\right]$. In other words, unlike the humans, the pigeons responded primarily on the basis of shape for the decomposable objects and primarily on the basis of motion for the nondecomposable objects.

New-motion and new-object tests. The results of the new-motion and new-object tests are shown in the two rightmost pairs of bars in the top and bottom panels of Figure 4 . These tests also revealed a difference between the decomposable and nondecomposable objects. The results of a repeated measures ANOVA with trial type, object type, and change type (new motion or new object) as factors are shown in Table 3, along with the effect sizes. Importantly, the three-way interaction was again significant, thus confirming the observation that the discrimination was differentially affected by the type of change for the nondecomposable objects and decomposable objects. 
Planned comparisons revealed that with the decomposable objects, the pigeons responded significantly more on go trials than on no-go trials on both the new-motion $\left[F(1,7)=35.82, M S_{\mathrm{e}}=0.038, \eta_{\mathrm{p}}^{2}=.837\right]$ and new-object $\left[F(1,7)=10.55, M S_{\mathrm{e}}=0.044, \eta_{\mathrm{p}}^{2}=.601\right]$ tests. This indicates that they had encoded both the shape and the motion of the stimuli. For the nondecomposable objects, the pigeons responded significantly more on go trials than on no-go trials on the new-object tests $[F(1,7)=26.05$, $\left.M S_{\mathrm{e}}=0.023, \eta_{\mathrm{p}}^{2}=.788\right]$, but not on the new-motion tests $[F(1,7)<1]$, indicating that they could not identify the shapes of the nondecomposable objects without their characteristic motion.

\section{DISCUSSION}

Our results provide clear evidence for a role of dynamic information in object recognition by both humans and pigeons. For both species, and for both easy- and hard-todecompose objects, reversing the characteristic motion of the two objects, or presenting them in a new motion, reduced discriminative responding based on object shape. However, the magnitude of the effect and its pattern across object types differed between species. Humans showed reductions in accuracy when motion information was changed, but they continued to respond primarily according to shape information with both types of objects. Pigeons, however, showed a large interaction between object type and motion. With decomposable objects, changes to the motion reduced the pigeons' discrimination performance, but the pigeons continued to respond primarily on the basis of shape. By contrast, with nondecomposable objects, the pigeons responded primarily on the basis of motion information: In particular, when the direction of motion was reversed, the tendency to respond go and no go also reversed, and when a new motion trajectory was presented, the birds showed no discrimination between the learned go and no-go shapes. A difference between species was also seen when the learned motions were carried by new objects: Pigeons showed good discrimination between the old $\mathrm{S}+$ and $\mathrm{S}-$ motions when they were carried by new objects of either type, whereas people did not respond significantly more to the old $\mathrm{S}+$ motion than to the old $\mathrm{S}-$ motion with new objects of either type.

That human performance decreased by approximately equal amounts when both decomposable and nondecomposable objects were displayed in the reverse- or newmotion conditions is a new finding. Vuong and Tarr (2006) had to degrade the same decomposable objects used in the present study with visual "dynamic fog" to obtain reliable decrements in performance on reverse-motion test trials. Further, the present comparison between decomposable and nondecomposable objects was within-subjects for the first time, and thus, the obtained differences in performance between stimulus types were not influenced by familiarity with the task.

There are two related explanations for why we observed a decrement in humans' performance with the decomposable objects undergoing reversed or new motions. First, the payoff scheme - rewarding fast and correct go responses more than correct no-go responses - may have encouraged participants to encode more cues, so that they could discriminate the $\mathrm{S}+$ object more quickly, especially if the cues are processed in parallel and responses are based on whichever cue is sufficient. Second, and more interesting, the present study presents the first time that human participants have been trained in a situation in which each novel object had its own characteristic motion (for a study on the role of characteristic facial motion, see Knappmeyer et al., 2003). Consequently, motion cues could be learned uniquely and could provide as good a basis for successful performance as could shape cues. Indeed, there is evidence that human observers are sensitive to and can learn unique motions produced by nonrigidly deforming spheres (Chuang et al., 2005). Together, these possibilities imply that the two-choice discrimination task that we used, with an incentive for fast and accurate responding and the possibility for motion cues alone to lead to successful performance, was a more sensitive measure of the effects of dynamic information on object recognition than was the four-choice identification task used in previous research (Vuong \& Tarr, 2006). Thus, the present study demonstrates that under certain circumstances, even visually distinctive shape information does not necessarily play the dominant role in object recognition for humans.

That the pigeons displayed a striking difference between the decomposable and nondecomposable objects is also a novel finding. Like humans, the pigeons appeared to be more sensitive to the shape of the decomposable objects than to the shape of the nondecomposable objects. With decomposable objects, the pigeons encoded both object shape and direction of motion and could use either cue to respond discriminatively. When the two cues were in conflict, the pigeons responded primarily on the basis of shape. In contrast, with nondecomposable objects, motion cues dominated the discrimination, and shape cues, by themselves, could not be used for discrimination. However, the shape cues apparently contributed to the discrimination of motion, because a change in the shape reduced discriminative responding.

Perhaps one of the most interesting results obtained with the pigeons is that they appeared to encode movement information somewhat independently of object information. At the very least, for the pigeons, motion information was weighed much more than shape information in their recognition of nondecomposable objects. Even when trained with decomposable objects, for which shape dominated responding on the conflict tests, they were able to use direction of movement alone to respond discriminatively to a novel object. This result is consistent with findings by Dittrich et al. (1998) on pigeons' ability to detect biological motion from points of light. They found not only that pigeons could learn to discriminate between pecking and walking movements in point-light displays, but also that pigeons trained to discriminate fully detailed video scenes of pecking versus walking showed some transfer of the discrimination to point-light scenes of the movement categories. Our results thus add to theirs 
in showing that pigeons can extract and respond on the basis of pure motion information from complex dynamic displays. The finding that pigeons were more sensitive than humans to the characteristic motion of nondecomposable objects is also interesting, especially in light of previous evidence suggesting that pigeons are less sensitive than humans in detecting coherent motion in random dot displays (Bischof et al., 1999). Whether pigeons are more sensitive to motion carried by objects than to motion in dot displays, or whether they are more sensitive to rotating motion than to linear motion is an interesting question for future research.

In the present study, the dynamic stimuli and training procedures were designed so that either shape or motion information could be used to discriminate between the $\mathrm{S}+$ and $\mathrm{S}-$. Further, the testing conditions were designed to tease apart the extent to which shape or motion cues were used by each species to perform the discrimination task. Under these circumstances, the intriguing finding here is that humans and pigeons weigh these cues differently (see Vuong \& Tarr, 2005, for a discussion of cue weighting in humans). There are numerous possible reasons for the species difference in weighting of shape and motion cues. For example, the difference in cue use may reflect differences in how the two different visual systems process shape and motion information (see, e.g., Bischof et al., 1999; Friedman et al., 2005; Spetch \& Friedman, 2003). Alternatively, the familiarity of the object might be a factor in the weighting given. Although the objects were unfamiliar to both humans and pigeons, it is possible that the decomposable objects were more similar to familiar objects for humans than they were for pigeons. If so, then perhaps motion is particularly relevant to pigeons when objects are unfamiliar. It is also possible that the species differences in weighting of shape and motion cues reflected differences in the procedures across species. Because discriminative shape information can be derived quickly, whereas discriminative motion information will take some time to accumulate, there is likely an inherent bias to attend to object shape. Our instructions to humans to respond quickly may have increased the bias toward responding according to shape. Nevertheless, it is interesting that both humans and pigeons used motion cues, even given this shape bias. Furthermore, for both species, this bias can be manipulated by changing the characteristics of the objects. These considerations strengthen the argument that motion is important for object recognition in both species.

This research is at an early stage, and more studies are needed in order to determine the generality of our results across modifications of the procedures and stimuli. The present study showed a species difference in the weighting of shape and motion cues when both cues were equally relevant during training. In future research, we will examine whether species differences also appear when only one cue, either motion or shape, is relevant and the other cue is irrelevant to the discrimination. It will also be interesting to determine whether the species differences hold if the characteristic motions of each object differ in their manner rather than in their direction of motion, or if more qualitatively different motions were used (e.g., rigid vs. nonrigid). We also wish to examine whether pigeons show the same results if directly viewing actual dynamic objects (see Friedman et al., 2005, for a recent study comparing recognition of objects seen directly with objects viewed in images for both humans and pigeons). Finally, we would like to explore pigeons' dynamic object recognition for more distantly viewed objects, in comparison with their recognition at the close viewing distances used in the present study. It is possible, for example, that the contribution of motion and shape information to object recognition will be different when pigeons view more distant objects using the lateral, monocular visual field than when viewing objects up close using frontal binocular vision.

The visual environments for humans and pigeons are undoubtedly very different. Consequently, the different weighting strategies that we observed in these species in the present study could reflect how each species processes its own particular environment and determines which visual cues from that environment to use for specific tasks, such as object recognition. But given that the environment for both humans and pigeons is dynamic, the results presented here underscore the ecological advantage of processing both shape and motion information for both species.

\section{REFERENCES}

Biederman, I. (1987). Recognition-by-components: A theory of human image understanding. Psychological Review, 94, 115-147.

Biederman, I., \& Gerhardstein, P. C. (1993). Recognizing depthrotated objects: Evidence and conditions for three-dimensional viewpoint invariance. Journal of Experimental Psychology: Human Perception \& Performance, 19, 1162-1182.

Bischof, W. F., Reid, S. L., Wylie, D. R. W., \& Spetch, M. L. (1999). Perception of coherent motion in random dot displays by pigeons and humans. Perception \& Psychophysics, 61, 1089-1101.

Blough, P. M. (2001). Cognitive strategies and foraging in pigeons. In R. G. Cook (Ed.), Avian visual cognition [Online]. Available at www .pigeon.psy.tufts.edu/avc/pblough.

Bülthoff, H. H., \& Edelman, S. (1992). Psychophysical support for a two-dimensional view interpolation theory of object recognition. Proceedings of the National Academy of Sciences, 89, 60-64.

Cavanagh, P., Labianca, A. T., \& Thornton, I. M. (2001). Attentionbased visual routines: Sprites. Cognition, 80, 47-60.

Chuang, L. L., Vuong, Q. C., Thornton, I. M., \& Bülthoff, H. H. (2005, August). Sequence selectivity of form transformation in visual object recognition. Poster presented at the 28th European Conference on Visual Perception, A Coruña, Spain.

Cook, R. G., \& Katz, J. S. (1999). Dynamic object perception by pigeons. Journal of Experimental Psychology: Animal Behavior Processes, 25, 194-210.

Cook, R. G., Shaw, R., \& Blaisdell, A. P. (2001). Dynamic object perception by pigeons: Discrimination of action in video presentations. Animal Cognition, 4, 137-146.

Dittrich, W. H., Lea, S. E. G., Barrett, J., \& Gurr, P. R. (1998). Categorization of natural movements by pigeons: Visual concept discrimination and biological motion. Journal of the Experimental Analysis of Behavior, 70, 281-299.

Edelman, S. (1999). Representation and recognition in vision. Cambridge, MA: MIT Press.

Friedman, A., Spetch, M. L., \& Ferrey, A. (2005). Recognition by humans and pigeons of novel views of 3-D objects and their photographs. Journal of Experimental Psychology: General, 134, 149-162.

Giese, M. A., \& Poggio, T. (2003). Neural mechanisms for the rec- 
ognition of biological movements. Nature Reviews Neuroscience, 4, 179-192.

Hayward, W. G., \& Williams, P. (2000). Viewpoint dependence and object discriminability. Psychological Science, 11, 7-12.

Husband, S., \& Shimizu, T. (2001). Evolution of the avian visual system. In R. G. Cook (Ed.), Avian visual cognition [Online]. Available at www.pigeon.psy.tufts.edu/avc/pblough.

Jitsumori, M., \& MAKINO, H. (2004). Recognition of static and dynamic images of depth-rotated human faces by pigeons. Learning \& Behavior, 32, 145-156.

Knappmeyer, B., Thornton, I. M., \& Bülthoff, H. H. (2003). The use of facial motion and facial form during the processing of identity. Vision Research, 43, 1921-1936.

LANDER, K., \& BRUCE, V. (2000). Recognizing famous faces: Exploring the benefits of facial motion. Ecological Psychology, 12, 259-272.

LIU, T., \& COOPER, L. A. (2003). Explicit and implicit memory for rotating objects. Journal of Experimental Psychology: Learning, Memory, \& Cognition, 29, 554-562.

Loftus, G. R., \& Masson, M. E. J. (1994). Using confidence intervals in within-subject designs. Psychonomic Bulletin \& Review, 1, 476490.

Newell, F. N., Wallraven, C., \& Huber, S. (2004). The role of characteristic motion in object categorization. Journal of Vision, 4, 118129.

Peissig, J. J., Wasserman, E. A., Young, M. E., \& Biederman, I. (2002). Learning an object from multiple views enhances its recognition in an orthogonal rotational axis in pigeons. Vision Research, $\mathbf{4 2}$, 2051-2062.

Spetch, M. L., \& Friedman, A. (2003). Recognizing rotated views of objects: Interpolation versus generalization by humans and pigeons. Psychonomic Bulletin \& Review, 10, 135-140.

Spetch, M. L., \& Friedman, A. (2006). Comparative cognition of object recognition. Comparative Cognition \& Behavior Reviews, 1, 12 35. Available at www.comparativecognition.org/.

Spetch, M. L., Friedman, A., \& Reid, S. L. (2001). The effect of distinctive parts on recognition of depth-rotated objects by pigeons (Columba livia) and humans. Journal of Experimental Psychology: General, 130, 238-255.
Stone, J. V. (1998). Object recognition using spatiotemporal signatures. Vision Research, 38, 947-951.

Stone, J. V. (1999). Object recognition: View-specificity and motionspecificity. Vision Research, 39, 4032-4044.

TARR, M. J., \& Pinker, S. (1989). Mental rotation and orientation dependence in shape recognition. Cognitive Psychology, 21, 233-282.

Thornton, I. M., \& Kourtzi, Z. (2002). A matching advantage for dynamic human faces. Perception, 31, 113-132.

Troje, N. F. (2002). Decomposing biological motion: A framework for analysis and synthesis of human gait patterns. Journal of Vision, $\mathbf{2}$, 371-387.

VuONG, Q. C., \& TARR, M. J. (2004). Rotation direction affects object recognition. Vision Research, 44, 1717-1730.

VuONG, Q. C., \& TARr, M. J. (2006). Structural similarity and spatiotemporal noise effects on learning dynamic novel objects. Perception, 35, 497-510.

Wallach, H., \& O'Connell, D. N. (1953). The kinetic depth effect. Journal of Experimental Psychology, 45, 205-217.

Wasserman, E. A., Gagliardi, J. L., Cook, B. R., KirkpatrickSteger, K., Astley, S. L., \& Biederman, I. (1996). The pigeon's recognition of drawings of depth-rotated stimuli. Journal of Experimental Psychology: Animal Behavior Processes, 22, 205-221.

ZeIGler, H. P., \& Bischof, H. J. (1993). Vision, brain, and behavior in birds. Cambridge, MA: MIT Press.

\section{NOTE}

1. Of the 21 participants whose data were included in the present analyses, three appeared not to have followed the speed instructions insofar as all their reaction times (RTs) on go trials were longer than the movie time; however, their responses were quite accurate. Four additional participants had few or no correct trials when the test motion was reversed from the training motion. If these participants were using motion as their sole cue to correct responding, then this pattern of incorrect responses is to be anticipated, but it precludes analyzing their RTs. We opted not to analyze the RT data in the present study, but rather, to include in our analyses of accuracy and proportion of go responses all participants who passed the accuracy criterion during training. 


\section{APPENDIX \\ Links to Animations of Objects Used in Training and Testing}

The animations were generated using the following functions to rotate each object about the $x$-axis, $y$-axis, and $z$-axis (in that order) by $\alpha^{\circ}$ from its initial fixed orientation on each time step:

$$
\begin{aligned}
& \alpha_{x}=360 \times \sin (t), \\
& \alpha_{y}=\frac{180}{\pi} \times t,
\end{aligned}
$$

and

$$
\alpha_{z}=360 \times \cos (t)
$$

where the parameter $t$ varies from 0 to $2 \pi$ for the training motion and $2 \pi$ to 0 for the new motion.

\section{Decomposable Objects}

Object A, Clockwise Motion: www.psych.ualberta.ca/ mspetch/ObjectReview/Pal06_m1.avi

Object A, Counterclockwise Motion: www.psych.ualberta.ca/ mspetch/ObjectReview/Pal06_m1R.avi

Object A, New Motion: www.psych.ualberta.ca/ mspetch/ObjectReview/Pa106_m2.avi

Object B, Clockwise Motion: www.psych.ualberta.ca/ mspetch/ObjectReview/Pal09_m1.avi

Object B, Counterclockwise Motion: www.psych.ualberta.ca/ mspetch/ObjectReview/Pa109_m1R.avi

Object B, New Motion: www.psych.ualberta.ca/ mspetch/ObjectReview/Pa109_m2.avi

Object C, Clockwise Motion: www.psych.ualberta.ca/ mspetch/ObjectReview/Pal21_m1.avi

Object C, Counterclockwise Motion: www.psych.ualberta.ca/ mspetch/ObjectReview/Pa121_m1R.avi

\section{Nondecomposable Objects}

Object A, Clockwise Motion: www.psych.ualberta.ca/ mspetch/ObjectReview/atom01_m1.avi

Object A, Counterclockwise Motion: www.psych.ualberta.ca/ mspetch/ObjectReview/atom01_m1R.avi

Object A, New Motion: www.psych.ualberta.ca/ mspetch/ObjectReview/atom01_m2.avi

Object B, Clockwise Motion: www.psych.ualberta.ca/ mspetch/ObjectReview/atom02_m1.avi

Object B, Counterclockwise Motion: www.psych.ualberta.ca/ mspetch/ObjectReview/atom02_m1R.avi

Object B, New Motion: www.psych.ualberta.ca/ mspetch/ObjectReview/atom02_m2.avi

Object C, Clockwise Motion: www.psych.ualberta.ca/ mspetch/ObjectReview/atom04_m1.avi

Object C, Counterclockwise Motion: www.psych.ualberta.ca/ mspetch/ObjectReview/atom04_m1R.avi

(Manuscript received August 31, 2005;

revision accepted for publication March 1, 2006.) 